\title{
A novel assay to diagnose hereditary angioedema utilizing inhibition of bradykinin-forming enzymes
}

\author{
K. Joseph ${ }^{1}$, S. Bains ${ }^{1}$, B. G. Tholanikunnel ${ }^{1}$, A. Bygum ${ }^{2}$, A. Aabom ${ }^{2}$, C. Koch ${ }^{2}$, H. Farkas ${ }^{3}$, L. Varga ${ }^{3}$, \\ B. Ghebrehiwet ${ }^{4} \&$ A. P. Kaplan ${ }^{1}$
}

\author{
${ }^{1}$ Medical university of South Carolina, Charleston, SC, USA; ${ }^{2}$ University of Southern Denmark and OPEN Odense Patient data Explorative \\ Network, Odense University Hospital, Odense, Denmark; ${ }^{3}$ 3rd Department of Internal Medicine, National Angioedema Center, Semmelweis \\ University, Budapest, Hungary; ${ }^{4}$ Stony Brook University, Stony Brook, NY, USA
} To cite this article: Joseph K, Bains S, Tholanikunnel BG, Bygum A, Aabom A, Koch C, Farkas H, Varga L, Ghebrehiwet B, Kaplan AP. A novel assay to diagnose
hereditary angioedema utilizing inhibition of bradykinin-forming enzymes. Allergy 2015; 70:115-119.

\section{Keywords}

bradykinin; factor XIla; hereditary angioedema; hereditary angioedema diagnosis; kallikrein.

\section{Correspondence \\ Kusumam Joseph, PhD, Medical University of South Carolina, Department of Biochem- istry and Molecular Biology, BSB- 501, 173 Ashley Avenue, Charleston, SC 29425, USA. Tel.: (843) 834-3339 \\ Fax: (843) 792-3200 \\ E-mail: josephk@musc.edu}

Accepted for publication 28 August 2014

DOI:10.1111/all.12520

Edited by: Thomas Bieber

\begin{abstract}
Background: Hereditary angioedema types I and II are caused by a functional deficiency of $\mathrm{C} 1$ inhibitor (C1-INH), leading to overproduction of bradykinin. The current functional diagnostic assays employ inhibition of activated $\mathrm{C} 1 \mathrm{~s}$; however, an alternative, more physiologic method is desirable.

Methods: ELISAs were developed using biotinylated activated factor XII (factor XIIa) or biotinylated kallikrein bound to avidin-coated plates. Incubation with plasma was followed by detection of bound C1-INH.

Results: After standard curves were developed for quantification of C1-INH, serial dilutions of normal plasma were employed to validate the ability to detect known concentration of $\mathrm{C} 1-\mathrm{INH}$ in the plasma as a percent of normal. Hereditary angioedema (HAE) types I and II were then tested. The level of functional C1-INH in all HAE types I and II plasma tested was less than $40 \%$ of our normal control. This was evident regardless of whether we measured factor XIIa-C1INH or kallikrein-C1-INH complexes, and the two assays were in close agreement. By contrast, testing the same samples utilizing the commercial method (complex ELISA, Quidel Corp.) revealed the levels of C1-INH between 0 and $57 \%$ of normal (mean, 38\%), and 42 samples were considered equivocal (four controls and 38 patients).

Conclusions: Diagnosis of HAE types I and II can be ascertained by inhibition of enzymes of the bradykinin-forming cascade, namely factor XIIa and kallikrein. Either method yields functional C1-INH levels in patients with HAE (types I and II) that are clearly abnormal with less variance or uncertainty than the commercial method.
\end{abstract}

Hereditary angioedema (HAE), types I and II, is an autosomal dominant disorder characterized by recurrent swelling in the extremities, face, gastrointestinal tract, or upper airways (1). Attacks last 2-5 days, and if not treated appropriately, swelling of the larynx, in particular, can be fatal. Since this is a rare disorder (affecting 1 in 20000 to 1 in 50000 people) with a variable presentation, the diagnosis may be missed.

\footnotetext{
Abbreviations

C1-INH, C1 inhibitor; HAE, hereditary angioedema; HK, high molecular weight kininogen.
}

hereditary angioedema is typically caused by a heterozygous mutation in the $\mathrm{Cl}$ inhibitor (C1-INH) gene, which results in either reduced protein levels (type I HAE, $85 \%$ of cases) or reduced function (type II HAE, 15\%) (2). To distinguish these two subtypes, the diagnosis of HAE is made by measuring both the antigenic and functional levels of C1-INH. In type I HAE, C1-INH protein level is low and the functional level is proportionately low, whereas in type II HAE, the protein level is normal, or even elevated, but the functional level is low. Thus, a functional assay is requisite, not only to confirm the diagnosis of HAE, but also type II disease cannot be diagnosed without it. Diagnosing HAE is critical as 
the mortality of untreated disease is around $30 \%$ due to asphyxiation (3-5).

C1 INH is a serine protease enzyme that inhibits activated proteins of the complement, coagulation, and kinin-forming cascades. The commercial assays used to assess C1-INH functional levels measure the inhibition of $\mathrm{C} 1 \mathrm{~s}$ of the complement cascade by C1-INH, utilizing either a chromogenic assay or a complex ELISA method. The chromogenic assay is generally considered preferable (6), but both methods have limitations. The chromogenic assay is more likely to have an occasional false positive while the complex ELISA has a negative predictive value of only $62 \%$ (6).

A theoretical limitation of both methods is that the assays measure the activity of C1-INH on an enzyme of the complement cascade and not the bradykinin-forming cascade. It is the inability of C1-INH to inhibit activated factor XII (7) and plasma kallikrein (8) that leads to overproduction of bradykinin, and the latter occurrence is the cause of angioedema in HAE (9). In support of this, a dysfunctional C1-INH has been described, which has normal activity on the kinin-forming cascade; hence, no angioedema occurs, but it fails to inhibit $\mathrm{C}$ 1s so that $\mathrm{C} 1$ is abnormally active and $\mathrm{C} 4$ is depleted $(10,11)$. If the reverse were to occur, that is, a mutated inhibitor that inhibits activated $\mathrm{C} 1 \mathrm{~s}$ but not kallikrein or factor XIIa, it could not be diagnosed with the current methodology. Thus, an assay with physiologic relevance would be a major advance over the current methods. We report herein two novel assays for functional C1-INH employing ELISA methodology based on either inhibition of activated factor XII or plasma kallikrein to diagnose HAE types I and II.

\section{Materials and methods}

\section{Patients and sample collection}

The diagnosis of HAE was confirmed by clinical presentation, low C1-INH protein, and/or functional level $(1,2)$ using the commercial assay performed at the site of origin of the samples. Citrated plasma from 87 patients (77 type I and 10 type II) with HAE and 47 healthy controls was separated by centrifugation of freshly collected blood at $1200 \mathrm{~g}$ for $10 \mathrm{~min}$ at $4^{\circ} \mathrm{C}$. All samples were immediately aliquoted and stored at $-80^{\circ} \mathrm{C}$. Samples were handled similarly at all participating sites (Odense, Denmark; Budapest, Hungary) and shipped overnight on dry ice. The protocol was approved by the Ethics Committee and Data Protection Agency at both participating sites.

Purified human factor XIIa and kallikrein were obtained from Enzyme Research Laboratories (South Bend, IN, USA), the biotinylation reagent was obtained from Thermo Scientific (Rockford, IL, USA), and all other reagents were obtained from Sigma Chemical Company (St. Louis, MO, USA).

\section{Biotinylation of proteins}

The proteins were biotinylated according to the manufacturer's recommendations. Briefly, $1 \mathrm{mg}$ of protein (kallikrein or factor XIIa) was dissolved in $0.5 \mathrm{ml}$ of phosphate-buffered saline (PBS). Twenty-seven microliters of freshly prepared $10 \mathrm{mM}$ Sulfo-NHS-LC-biotin was added to the protein solution and incubated on ice for $2 \mathrm{~h}$. Excess nonreacted and hydrolyzed biotin was removed using a spin-desalting column. The labeling of the proteins was confirmed by ELISA, and the protein concentration was determined by Bradford assay (12).

\section{ELISA to measure C1-INH protein level in plasma}

Immulon $2 \mathrm{HB}$ plates were coated with $5 \mu \mathrm{g} / \mathrm{ml}$ polyclonal antibody to C1-INH. After blocking with $1 \%$ BSA in PBS, samples and standards were added and incubated at room temperature for $1 \mathrm{~h}$. Bound $\mathrm{C} 1-\mathrm{INH}$ was probed with alkaline phosphatase-conjugated monoclonal antibody to C1-INH followed by color development using 5-bromo-4chloroindolyl phosphate/nitroblue tetrazolium (BCIP/NBT).

\section{Quantification of functional C1-INH in plasma based on inhibition of kallikrein and factor XIIa}

Immulon $2 \mathrm{HB}$ plates were coated with $5 \mu \mathrm{g} / \mathrm{ml}$ avidin in coating buffer $(100 \mu \mathrm{l})$ overnight at $4^{\circ} \mathrm{C}$. Plates were washed three times using PBS-Tween (200 $\mu \mathrm{l} / \mathrm{each})$. Subsequently, $200 \mu 1 \%$ BSA in PBS was added to block the unused sites. The plates were incubated at $37^{\circ} \mathrm{C}$ for $1 \mathrm{~h}$ and were washed three times using PBS-Tween $(200 \mu \mathrm{l} / \mathrm{each})$. Samples or standards were added to the plates along with biotinylated protein $(25 \mu \mathrm{l}$ standards or samples, $25 \mu \mathrm{l}$ biotinylated factor XII or biotinylated kallikrein $(1 \mu \mathrm{g} / \mathrm{ml})$, and $50 \mu \mathrm{l}$ binding buffer), were mixed, and incubated at $37^{\circ} \mathrm{C}$ for $1 \mathrm{~h}$. After incubation, plates were washed three times using PBS-Tween $(200 \mu \mathrm{l} / \mathrm{each})$. A polyclonal antibody to $\mathrm{C} 1-\mathrm{INH}$ was added, followed by incubation at room temperature for $1 \mathrm{~h}$. The wells were washed with PBS-Tween three times. Alkaline phosphatase-conjugated secondary antibody was added, and the samples were incubated at room temperature for $1 \mathrm{~h}$ followed by color development using phosphatase substrate $\mathrm{BCIP} / \mathrm{NBT}$. The OD at $450 \mathrm{~nm}$ was read, and calculations were performed using the standard curve. The method is summarized step by step in Fig. 1.

\section{Quantification of functional ELISA based on inhibition of C1s}

ELISA kit was purchased from Quidel Corporation to measure the amount of functional C1-INH. This assay is based on the ability of plasma C1-INH to inhibit the first component of the complement cascade: activated C1s (utilizing a complex ELISA assay). The assay was performed according to the manufacturer's protocol.

\section{Results}

Diagnosis of HAE by inhibition of C1s

We tested C1 INH function of 46 samples from normal controls and 87 samples from patients with either type I or type II 


\section{ELISA method to detect functional C1-INH in plasma}

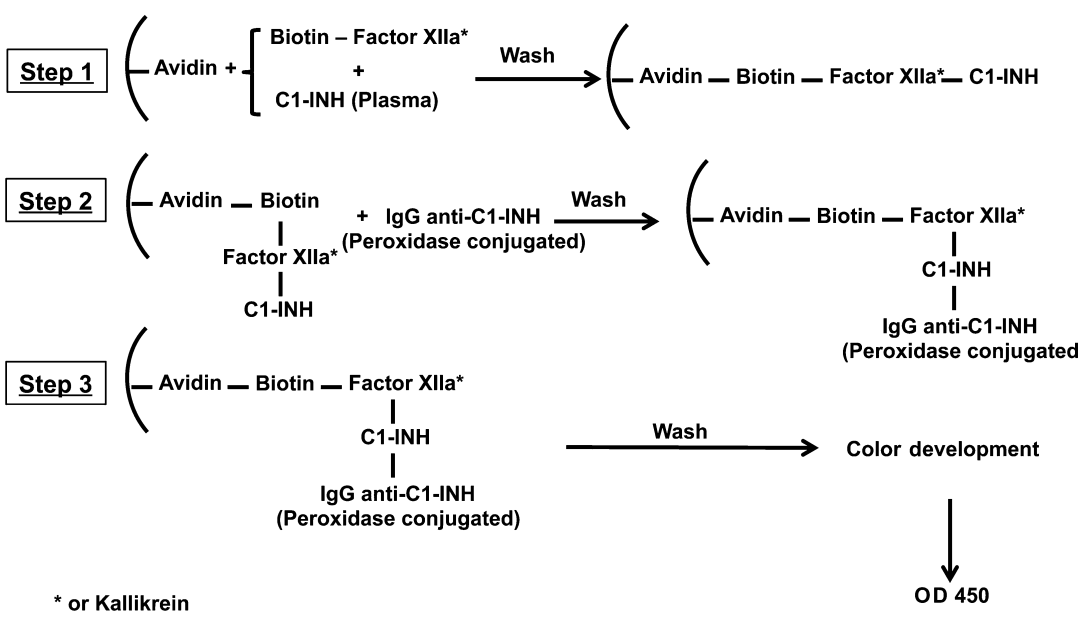

Figure 1 A diagrammatic representation of the ELISA method to detect functional C1-INH in plasma.

HAE using a commercial assay (complex ELISA, Fig. 2A). According to the assay interpretation, 'normal' is $68-100 \%$ C1-INH while 'abnormal' is below $67 \%$. The instructions indicate that samples between $41 \%$ and $67 \%$ are to be repeated, because they are considered equivocal, but if the repeat value is within this range, it is reported as abnormal. Standards were supplied as a percentage of normal, that is, standards were $0 \%, 23 \%, 44 \%, 66 \%$, and $88 \%$, and unknowns are read off the curve. The normal subjects varied between $62 \%$ and $100 \%$ while the HAE samples varied between 0 and $57 \%$. The mean and standard deviation for HAE samples was $38 \pm 13 \%$. There were 42 samples that were considered equivocal (four controls and 38 patients) and needed repeat measurements.
The repeat measurements revealed normal levels in controls and were abnormal in the patients with HAE.

\section{Diagnosis of HAE by inhibition of kallikrein or factor XIIa}

The results employing inhibition of plasma kallikrein or inhibition of factor XIIa expressed as percentage of control (pooled normal plasma) are shown in Fig $2 \mathrm{~B}$ and $\mathrm{C}$, respectively, where normal controls are compared to samples obtained from the same patients with types I and II HAE assayed above. The mean and standard deviation for factor XIIa-C1-INH were as follows: normal, $90 \pm 12 \%$; types I and II HAE, $16 \pm 8 \%$. The ' $P$ ' value comparing types I and

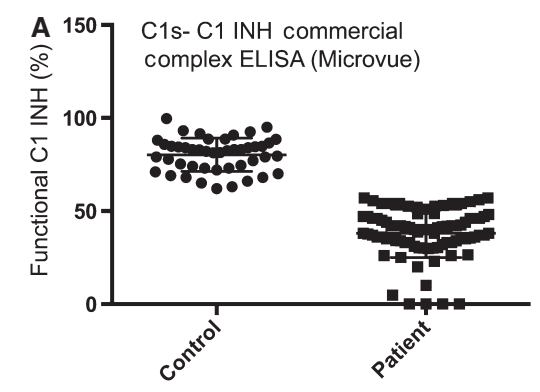

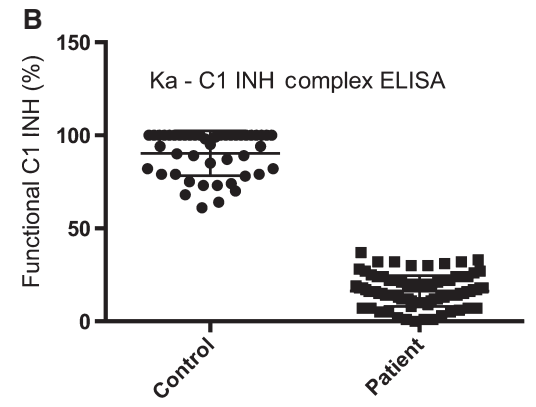

Figure 2 Functional ELISA based on inhibition of complement C1s (A), kallikrein (B), or factor XIla (C): ELISAs were performed as

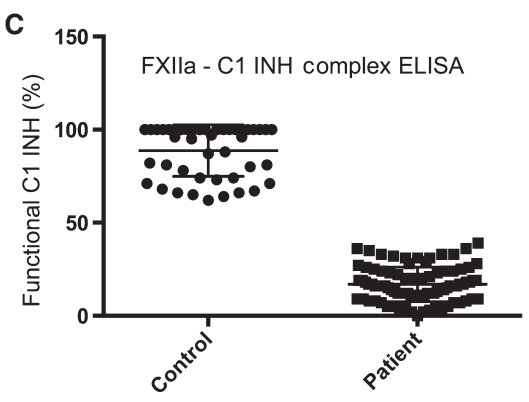

described in 'Materials and methods'. Results (mean \pm SD) are expressed as percentage of controls. 
II HAE to normal controls was $<0.0001$. The results obtained assaying for kallikrein-C1-INH complexes were strikingly similar with no overlap between groups (normal, $89 \pm 14 \%$; types I and II HAE, $17 \pm 9 \%$; 'P' value comparing types I and II HAE to normal controls: <0.0001). There were no false positives or false negatives employing this methodology. These assays were performed in duplicate.

\section{Discussion}

Our results show that the assays for factor XIIa-C1-INH complex formation or for kallikrein-C1-INH complex formation correctly identified $100 \%$ of the patients with types I and II HAE with low functional C1-INH as compared to normal controls (Figs 1 and 2). Our rationale was that it would be preferable to develop an assay that measures the activity of C1-INH on an enzyme that is requisite for bradykinin formation. These would therefore employ one of the activated forms of factor XII (factor XIIa or factor XIIf) or plasma kallikrein. We assumed that inhibition of factor XIIa would be simpler since it has no other significant inhibitor in plasma while plasma kallikrein is also inhibited by $\alpha 2$ macroglobulin. Nevertheless, both methods work well as we detect only that fraction of kallikrein $(\sim 60 \%)$, which is inhibited by C1-INH (13).

Our approach was to biotinylate the active enzyme, bind it to an avidin-coated plate, incubate with plasma (normal for a control, putative HAE plasma as the unknowns), and measure the enzyme-C1-INH complex. We employed alkaline phosphatase-labeled antibody to C1-INH for the detection of bound C1-INH. For the quantification of C1-INH, a standard curve was made by substituting known quantities of C1-INH in buffer, in place of plasma. This method is similar to the complex ELISA method currently employed for inhibition of C1s by C1-INH (14). Furthermore, if other types of angioedema (HAE with normal C1-INH function, for example) had a mutant C1-INH that inhibits activated factor XII or kallikrein but not $\mathrm{C} 1 \mathrm{~s}$, we would detect the abnormality using this method, whereas the diagnosis would be missed employing either of the currently available assays. Our methods have the possibility to supplant the current commercial methods for the diagnosis of types I and II HAE as either one appears to be more sensitive for the detection of dysfunctional C1-INH than does the inhibition of $\mathrm{C} 1 \mathrm{~s}$, and/or could also be used to evaluate patients where an equivocal result is obtained employing current methodology. The assay is identical in complexity to any of the commercial assays and can be performed in $3-4 \mathrm{~h}$ if it is made available commercially. The diagnosis of HAE can be made with these assays alone, although a C1-INH protein determination is needed to distinguish type II HAE from type I HAE. It can also shed further light on type III HAE, that is, HAE with 'normal' $\mathrm{C} 1$ inhibitor and readily distinguish it from type I or II HAE. About $5 \%$ of patients with type I or II HAE have a normal C4 level when asymptomatic (2), even though functional C1-INH is abnormal; during an attack of swelling, $\mathrm{C} 4$ approaches zero and levels of C2 decline (15). Thus, in patients with type II HAE, the diagnosis can be dependent solely on a functional assay unless a mutation in C1-INH protein or mRNA is defined. Our method(s) would be particularly useful in this circumstance. In conclusion, the diagnosis of HAE types I and II can be ascertained by inhibition of enzymes of the bradykinin-forming cascade so that the diagnosis is made by functional assessment that is directly related to the abnormality leading to angioedema.

\section{Author contributions}

Kusumam Joseph, PhD, Sonia Bains, MD, and Baby Tholanikunnel, $\mathrm{PhD}$, were responsible for planning, execution of experiments, data analysis, and manuscript preparation; Anette Bygum, MD, Anne Aabom, MD, Claus Koch, PhD, Henriette Farkas, MD, and Lilian Varga, PhD, have collected HAE samples according to our instructions; Berhane Ghebrehiwet, DVM, prepared the antisera used in the studies; Allen Kaplan, MD, was involved with planning, data analysis, and manuscript preparation.

\section{Funding}

The study received funds from Dyax Corp., 55 Network Drive, Burlington, MA 01803.

\section{Conflicts of interest}

Kusumam Joseph has research grants from Dyax Corp., CSL Behring, and Shire Human Genetic Therapies Inc. Anette Bygum has been involved in clinical research or educational events involving CSL Behring, Shire, Sobi, and ViroPharma. Henriette Farkas is a member of the Advisory Board of CSL Behring, Shire Human Genetic Therapies Inc., and Pharming, also received speaker fee from Swedish Orphan Biovitrum. Allen Kaplan is involved as Speakers Bureau with Dyax Corp., Shire, and ViroPharma.

\section{References}

1. Frank MM, Gelfand JA, Atkinson JP. Hereditary angioedema: the clinical syndrome and its management. Ann Intern Med 1976;84:580-593.

2. Zuraw B. Clinical practice. Hereditary angioedema. N Engl J Med 2008;359:1027-1036.

3. Agostoni A, Cicardi M. Hereditary and acquired $\mathrm{Cl}$-inhibitor deficiency: biological and clinical characteristics in 235 patients. Medicine (Baltimore) 1992;71:206-215.

4. Bork K, Siedlecki K, Bosch S, Schopf RE, Kreuz W. Asphyxiation by laryngeal edema in patients with hereditary angioedema. Mayo Clin Proc 2000;75:349-354.

5. Bork K, Hardt J, Witzke G. Fatal laryngeal attacks and mortality in hereditary angioe- dema due to C1-INH deficiency. $J$ Allergy Clin Immunol 2012;130:692-697.

6. Wagenaar-Bos IGA, Drouet C, AygorenPursun E, Bork K, Bucher C, Bygum A, et al. Functional C1-inhibitor diagnostics in hereditary angioedema: assay evaluation and recommendations. J Immunol Methods 2008;338:14-20. 
7. Kaplan AP, Joseph K. The bradykinin-forming cascade and its role in hereditary angioedema. Ann Allergy Asthma Immunol 2010; 104:193-204.

8. Gigli I, Mason JW, Colman RW, Austen KF. Interaction of plasma kallikrein with the C1 inhibitor. J Immunol 1970;104:574581.

9. Kaplan AP, Joseph K. Kinin formation in C1 inhibitor deficiency. J Allergy Clin Immunol 2010;125:1411-1412.

10. Zahedi R, Bissler JJ, Davis AE III, Andreadis C, Wisnieski JJ. Unique C1 inhibitor dysfunction in a kindred without angioedema: II. Identification of an Ala443-Val substi- tution and functional analysis of the recombinant mutant protein. $J$ Clin Invest 1995;95:1299-1305.

11. Wisnieski JJ, Knauss TC, Yike I, Dearborn DG, Narvy RL, Naff GB. Unique C1 inhibitor dysfunction in a kindred without angioedema. I. A mutant $\mathrm{cl}$ inhibitor that inhibits C1s but not C1r. J Immunol 1994;152:31993209.

12. Bradford MM. A rapid and sensitive method for the quantitation of microgram quantities of protein utilizing the principle of proteindye binding. Anal Biochem 1976;72:248-254.

13. Harpel PC, Lewin MF, Kaplan AP. Distribution of plasma kallikrein between C-1 in- activator and alpha 2-macroglobulin in plasma utilizing a new assay for alpha 2macroglobulin-kallikrein complexes. $J$ Biol Chem 1985;260:4257-4263.

14. Tarzi MD, Hickey A, Forster T, Mohammadi M, Longhurst HJ. An evaluation of tests used for the diagnosis and monitoring of $\mathrm{C} 1$ inhibitor deficiency: normal serum $\mathrm{C} 4$ does not exclude hereditary angio-oedema. Clin Exp Immunol 2007;149:513-516.

15. Austen KF, Sheffer AL. Detection of hereditary angioneurotic edema by demonstration of a reduction in the second component of complement. N Eng J Med 1965;272:649656. 